\title{
Silver Nitrate
}

National Cancer Institute

\section{Source}

National Cancer Institute. Silver Nitrate. NCI Thesaurus. Code C77057.

An inorganic chemical with antiseptic activity. Silver nitrate can potentially be used as a cauterizing or sclerosing agent. 